\title{
Stacked Deep Inferior Epigastric Perforator Free Flaps with Immediate Implant Placement for Unilateral Breast Reconstruction
}

\author{
Keith C. Hood, MD ${ }^{1} \quad$ Nishant Ganesh Kumar, BS ${ }^{1} \quad$ Stephane A. Braun, MD ${ }^{1}$ Kent Kye Higdon, MD ${ }^{1}$ \\ ${ }^{1}$ Department of Plastic Surgery, Vanderbilt University Medical \\ Center, Nashville, Tennessee \\ J Reconstr Microsurg Open 2017;2:e72-e74. \\ Address for correspondence Keith C. Hood, MD, Department of \\ Plastic Surgery, Vanderbilt University Medical Center, D-4207 Medical \\ Center North, Nashville, TN 37232 \\ (e-mail: keith.c.hood@vanderbilt.edu).
}

\begin{abstract}
Keywords

- stacked DIEP flap

- free flap with immediate implant

- breast reconstruction
\end{abstract}

A variety of breast reconstruction techniques exist for patients undergoing mastectomy for breast cancer treatment, including implant-based reconstruction and autologous tissue reconstruction. This covers a range of options such as latissimus dorsi flaps, transverse rectus abdominus flaps (free and muscle sparing), deep inferior epigastric artery perforator flaps, and gluteal artery perforator flaps. ${ }^{1,2}$ In achieving the ideal reconstructive and aesthetic result, techniques have been developed that combine autologous tissue with implant-based reconstruction. ${ }^{3-5}$

In some patients with inadequate abdominal tissue volume for breast reconstruction, a stacked DIEP flap can be used that incorporates the full soft tissue volume of both sides of the abdomen with their respective deep inferior epigastric perforating vessels. ${ }^{6}$ Herein, we discuss our experience with combining an implant with a stacked DIEP flap, which has yet to be reported, in a woman with prior surgical- and radiation-treated breast cancer.

received

April 18, 2017

accepted after revision

May 25, 2017
DOI https://doi.org/

10.1055/s-0037-1604155. ISSN 2377-0813.

\section{Methods/Results}

\section{Preoperative Evaluation}

The patient was a 53-year-old female patient with a body mass index (BMI) of $27.4(81.6 \mathrm{~kg})$ who presented to Vanderbilt University Medical Center for autologous left breast reconstruction secondary to pain and asymmetry. On examination, she had a Baker grade 3 capsular contracture with cephalic migration of the implant (-Fig. 1). Her history included bilateral subpectoral saline implants placed approximately 15 years ago. She subsequently developed left breast cancer that was treated with breast conservation therapy and radiation in 2010. She had since then developed recurrent left capsular contracture requiring two revisionary surgeries with capsulectomies and implant removal and replacement. The patient was a former smoker who quit in January 2016.
Copyright $\odot 2017$ by Thieme Medical Publishers, Inc., 333 Seventh Avenue, New York, NY 10001, USA. Tel: +1(212) 584-4662.
License terms

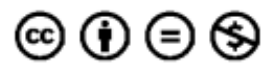




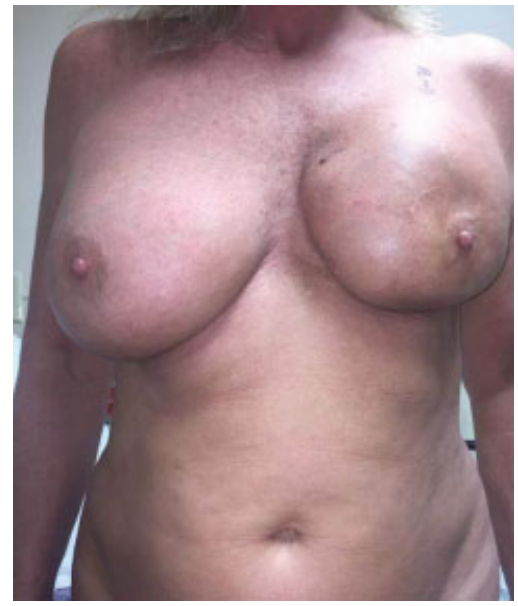

Fig. 1 The patient was evaluated preoperatively and found to have left breast Baker grade 3 capsular contracture with cephalic migration of the implant.

\section{Operative Technique}

The patient was marked and the flap designed preoperatively in the standing position. The previous left mastectomy scar was excised, a total capsulectomy was performed, and an intact implant was removed from the submuscular plane. The pectoralis major was transposed back to the chest wall and the third rib cartilage was identified facilitating exposure of the underlying internal mammary artery and vein.

The bilateral DIEP flaps were meticulously dissected and a single artery and vein were identified at their respective takeoffs and ligated to free the flap bilaterally. A 5,000 unit IV heparin bolus was given prior to ligation and allowed to circulate for 3 minutes, and the flap was flushed with heparinized PlasmaLyte after division.

Each hemiabdominal perforator flap was anastomosed separately to the internal mammary system in an antegrade and retrograde fashion. Venous anastomosis was completed using a 3.0 venous coupler, and interrupted 9-0 nylon sutures were utilized for the arterial anastomosis. A volumetric asymmetry was identified and to correct this, a Mentor (Irvine, CA)
$190 \mathrm{~L}$ round smooth silicone implant was placed under the flap. The pedicles were reinspected and there was no evidence of any tension, torsion, or kinking. Areas of the flap were deepithelialized and inset with 3-0 Vicryl deep dermal interrupted sutures and a running 4-0 Monoderm Quill suture. Surgical glue was placed over the incisions and both flaps had excellent flow as determined by hand-held Doppler at the end of the case.

Postoperatively, she recovered well with no complications and was discharged on postoperative day 3. Her 6-month postoperative results are shown ( $\mathbf{- F i g . ~} \mathbf{2 A - C}$ ).

\section{Discussion}

This is the first report of using both hemiabdominal flaps for autologous unilateral breast reconstruction while utilizing a prosthesis simultaneously. In a review on tertiary breast reconstruction, Baker grade 3 or 4 capsular contracture (followed by asymmetry) was the most common indication for autologous reconstruction in patients who had failed or had unsatisfactory results from implant-based reconstruction. The DIEP flap is often the predominant choice for autologous tertiary reconstruction. ${ }^{7}$ While the surgery is more technically demanding, the DIEP flap has the advantage of less donor-site morbidity compared with latissimus dorsi (LD) or transverse rectus abdominis (TRAM) flap. The stacked free DIEP flap can provide the soft tissue volume that a LD, TRAM, inferior gluteal artery perforator (GAP) flap, or a transverse upper gracilis (TUG) flap often cannot offer.

With a history of recurrent capsular contracture requiring a total capsulectomy and explantation in the submuscular plane, we felt a prepectoral plane would be ideal for implant placement. To ensure safety of the vascular pedicles, it was meticulously positioned to avoid kinking. A round smooth device was chosen to avoid the complication of adherence seen in textured implants or a possible rotational problem with shaped implants. This technique to ensure pedicle safety has also been described, ${ }^{8}$ and therefore, we felt confident in our choice. A delayed approach would require redissection of the prepectoral place where our vascular

A
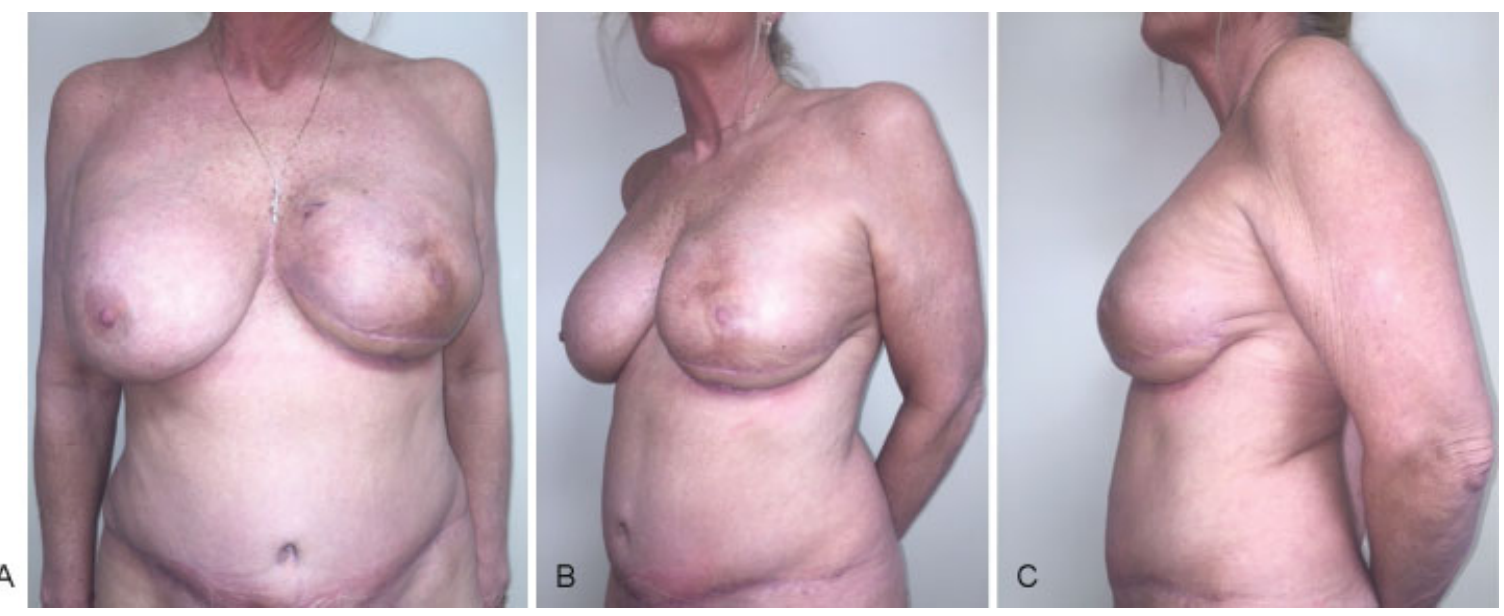

Fig. 2 (A-C) The patient's 6-month postoperative results are shown. 
pedicles resided. This was discussed and immediate implant placement was deemed the safest choice for the patient.

The use of an implant to enhance the volume of a reconstructed breast with a DIEP flap, both in the immediate and delayed setting, has been previously reported. Figus et al showed promising results as a safe and effective technique with high patient satisfaction. ${ }^{8}$ However, in their study, the effects of radiation were not investigated. Chang et al studied the impact of radiation on a series of flaps, including DIEP flaps. They found that there was no significant difference in complication rates or revisionary surgeries based on the type of flap used or if the patient had radiation therapy. However, the effect on implants was not included. ${ }^{9}$ Literature describing the impact of radiation on implant-based breast reconstruction in conjunction with free tissue transfer is still evolving.

Another option for our patient would have been exclusive autologous reconstruction with the addition of fat grafting. However, future revisionary surgeries would be a certainty to achieve the same result as an implant. Lipofilling alone without addition of a single or stacked flap could not have likely achieved the desired reconstructive and aesthetic result.

In light of our patient's history involving radiation and prior implant placement and the existing literature on tertiary breast reconstruction with implants, our outcome in this case report suggests that the stacked DIEP with an implant may serve as a promising technique in the armamentarium of the reconstructive surgeon.
Conflict of Interest

None.

\section{References}

1 Shons AR, Mosiello G. Postmastectomy breast reconstruction: current techniques. Cancer Contr 2001;8(05):419-426

2 Tseng CY, Lipa JE. Perforator flaps in breast reconstruction. Clin Plast Surg 2010;37(04):641-654, vi-ii

3 Healy C, Allen RJ Sr. The evolution of perforator flap breast reconstruction: twenty years after the first DIEP flap. J Reconstr Microsurg 2014;30(02):121-125

4 Kronowitz SJ, Robb GL, Youssef A, et al. Optimizing autologous breast reconstruction in thin patients. Plast Reconstr Surg 2003; 112(07):1768-1778

5 Trignano E, Fallico N, Dessy LA, et al. Transverse upper gracilis flap with implant in postmastectomy breast reconstruction: a case report. Microsurgery 2014;34(02):149-152

6 DellaCroce FJ, Sullivan S-K, Trahan C. Stacked deep inferior epigastric perforator flap breast reconstruction: a review of 110 flaps in 55 cases over 3 years. Plast Reconstr Surg 2011; 127(03):1093-1099

7 Mohan AT, Al-Ajam Y, Mosahebi A. Trends in tertiary breast reconstruction: literature review and single centre experience. Breast 2013;22(02):173-178

8 Figus A, Canu V, Iwuagwu FC, Ramakrishnan V. DIEP flap with implant: a further option in optimising breast reconstruction. J Plast Reconstr Aesthet Surg 2009;62(09):1118-1126

9 Chang EI, Liu TS, Festekjian JH, Da Lio AL, Crisera CA. Effects of radiation therapy for breast cancer based on type of free flap reconstruction. Plast Reconstr Surg 2013;131(01):1e-8e 